\title{
Téoros
}

Revue de recherche en tourisme

\section{Le Transsibérien, vecteur de la mise en tourisme des destinations enclavées}

\section{Le cas de la Russie}

\section{Ekaterina Andreeva-Jourdain}

Volume 32, numéro 2, 2013

Tourisme et transport

URI : https://id.erudit.org/iderudit/1036592ar

DOI : https://doi.org/10.7202/1036592ar

Aller au sommaire du numéro

Éditeur(s)

Université du Québec à Montréal

ISSN

0712-8657 (imprimé)

1923-2705 (numérique)

Découvrir la revue

Citer cet article

Andreeva-Jourdain, E. (2013). Le Transsibérien, vecteur de la mise en tourisme des destinations enclavées : le cas de la Russie. Téoros, 32(2), 26-36.

https://doi.org/10.7202/1036592ar
Résumé de l'article

Le rôle du train dans le développement des nouvelles destinations constitue la question centrale de cette étude. Cet article analyse l'importance du Transsibérien dans le développement territorial des lieux qu'il traverse avec un intérêt particulier porté sur le tourisme à l'échelle d'un pays comme la Russie. L'étude apporte un certain nombre de réponses historiques et géographiques avec un accent singulier sur l'importance et les différentes formes d'interactions possibles entre le train et le territoire. L'utilisation de la méthode de récolte de données basée sur l'analyse des catalogues des voyagistes cherche à identifier l'importance des lieux du point de vue touristique sur les parcours des trains permettant de produire des données fiables concernant le Transsibérien. 


\title{
Le Transsibérien, vecteur de la mise en tourisme des destinations enclavées Le cas de la Russie
}

\author{
Ekaterina ANDREEVA-JOURDAIN \\ Docteure en géographie et histoire \\ Espace et sociétés (ESO) Angers \\ ekaterina.jourdain@univ-angers.fr
}

\begin{abstract}
RÉSUMÉ: Le rôle du train dans le développement des nouvelles destinations constitue la question centrale de cette étude. Cet article analyse l'importance du Transsibérien dans le développement territorial des lieux qu'il traverse avec un intérêt particulier porté sur le tourisme à l'échelle d'un pays comme la Russie. L'étude apporte un certain nombre de réponses historiques et géographiques avec un accent singulier sur l'importance et les différentes formes d'interactions possibles entre le train et le territoire. L'utilisation de la méthode de récolte de données basée sur l'analyse des catalogues des voyagistes cherche à identifier l'importance des lieux du point de vue touristique sur les parcours des trains permettant de produire des données fiables concernant le Transsibérien.
\end{abstract}

Mots-clés : train, transport, Transsibérien, destination, Russie, tourisme, Etat.

Le développement du tourisme en Russie est possible grâce aux transformations sociales et spatiales que connaît le pays depuis la chute de l'Union Soviétique en 1991. Depuis la fin du régime communiste, les grandes villes russes se trouvent au centre de transformations économiques et sociales, avec l'affirmation d'une société de consommation associée à une sphère des loisirs. Depuis le début des années 1990, les touristes étrangers et russes réinvestissent les régions touristiques présentant un intérêt historique et culturel, comme les villes de Moscou, de Saint-Pétersbourg et celles de l'Anneau d'Or (Vladimir, Souzdal, Iaroslavl, etc.) L'organisation de voyages portant le nom «Transsibérien» destinés principalement à la clientèle étrangère commence après la chute du Rideau de fer au début des années 1990 (Andreeva-Jourdain, 2014).

Le Transsibérien n'est pas seulement une voie reliant Moscou à l'Asie orientale sur 9288 kilomètres ou une ligne traversant 20 entités régionales, 5 régions et plus de 990 gares parmi lesquelles se trouvent de grandes villes comme Iaroslavl, Ekaterinbourg, Novossibirsk, Krasnoïarsk, OulanBator, Khabarovsk, Pékin, Vladivostok (Chemins de fer russes, 2013). Le Transsibérien est un élément important de l'économie de la Russie, de son système de transport, ainsi qu'un acteur important de l'histoire du pays. Ce chemin de fer représente aussi une particularité unique en son genre du point de vue touristique. L'appellation «Transsibérien» porte souvent à une certaine confusion. Contrairement aux idées reçues, il n'existe pas un seul train ou une seule voie ferrée traversant la Sibérie (Chemins de fer russes, 2013). L'offre touristique «transsibérienne» représente différents produits et différents trajets (illustration 1):

- le Rossiâ (train numéro 2) et le train numéro 100E, parcourant les 9288 km reliant Moscou à Vladivostok;

- le Transmongolien, reliant Moscou à Pékin via la capitale mongole Oulan-Bator;

- le Transmandchourien, reliant également Moscou à Pékin, mais par Harbin, en Chine.

Le train quotidien qui s'arrête dans de nombreuses villes et que l'on nomme «Transsibérien» (Transsib, ou Transsibirskaâ Magistral', soit Grande Route Transsibérienne) correspond à la ligne de chemin de fer et non aux différents trains. Contrairement à ce premier type de train, le «Grand Transsibérien Express» ou «l'Or des Tsars Zarengold» ou encore le «Golden Eagle» sont réellement des produits de luxe correspondant à un itinéraire bien précis. Ce type de produit touristique intéresse majoritairement les touristes internationaux.

Depuis son existence, ce chemin de fer transsibérien a suscité de l'intérêt scientifique. Différents travaux portent 


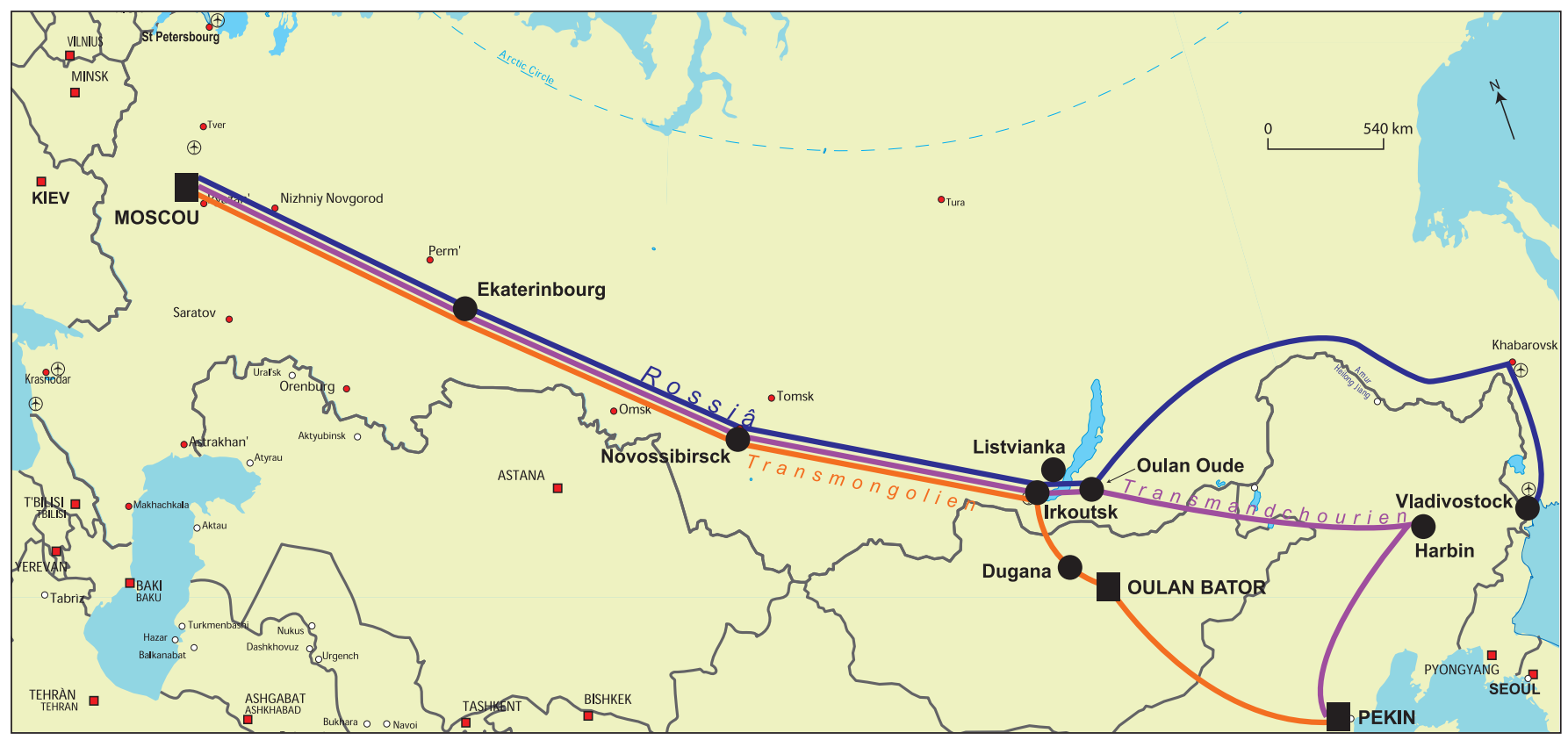

ILLUSTRATION 1 : Les parcours du Transsibérien et leurs principales étapes (source : conception de l'auteure).

sur l'histoire de la construction de la ligne et de son évolution dans le temps (Senin, 2001; Remencov, 2003; Mosse, 2001). Les ouvrages russes font souvent appel à l'importance économique et stratégique de ce chemin de fer pour la Russie en étudiant principalement son rôle dans la communication entre la partie européenne du pays et l'Asie orientale (Durakov, 2007; Uhtomin, 2005). Il existe également un grand nombre de récits en français (Bellec, 2008; Dunbar, 2006; Thomas, 2005) consacrés au voyage à bord de ce train qui contribuent à la popularité de ce produit touristique parmi la clientèle française, francophone et francophile. Néanmoins, ces études consacrées au Transsibérien en tant que moyen de transport ou en tant que train-légende négligent souvent son rôle dans le développement des nouvelles destinations touristiques. Comme nous le verrons dans cet article, le Transsibérien est un élément fondamental dans le cadre de l'étude d'un pays comme la Russie : il relie la Russie européenne à l'Asie orientale. De ce fait, le train a créé un certain nombre de lieux touristiques et continue à le faire.

Cet article a pour objectif de comprendre et de démystifier le rôle du train transsibérien dans le développement des destinations touristiques qu'il traverse. Un accent particulier sera porté aux territoires russes. À partir d'un certain nombre de réponses historiques et géographiques obtenues par le biais d'une lecture plus générale des éléments sur la manière dont les territoires russes traversés par le Transsibérien se positionnent aujourd'hui dans le champ du tourisme, cette analyse apporte un éclairage sur le fonctionnement touristique en Russie.

Le manque de statistiques fiables concernant le nombre de déplacements touristiques effectués à bord de ces différents trains a amené l'auteure à faire appel à la méthode de production des données proposée par Philippe Violier (2011). Cette méthode est fondée sur l'analyse des catalogues des voyagistes et sera présentée dans la deuxième partie de cet article. L'utilisation de cette méthode a permis d'identifier les lieux pour les deux études de cas présentées en troisième partie. Ils reflètent les différents types d'évolution des caractéristiques des territoires traversés par le train. La méthodologie ici retenue repose également sur un corpus qui se compose de documents officiels (textes de lois consacrés aux projets d'État russes en termes de développement touristique et de transport ferroviaire) et des archives d'État. Enfin, l'étude s'appuie également sur l'analyse de textes de la presse russe reflétant bien la situation actuelle en matière de l'aménagement touristique des territoires étudiés.

Dans cette perspective, après une présentation du train transsibérien comme un moyen de transport touristique, notre attention sera portée sur l'analyse des lieux qu'il traverse, suivie par une étude d'évolution de leurs caractéristiques à travers des cas précis.

\section{Le Transsibérien, un moyen de transport touristique}

Les machines à vapeur ont longtemps fasciné et fascinent toujours encore : la locomotive peut être «mise en avant comme un objet d'intérêt pour le voyageur» (Polino, 2007 : 29). Le train joue en rôle important dans un pays comme la Russie. Le réseau ferroviaire russe est plus développé et plus sûr que les lignes aériennes. À la différence de l'avion, le train permet aux voyageurs de se mettre en mouvement lent afin d'obtenir une expérience dépaysante forte. L'attractivité des trains touristiques se base également sur la beauté des paysages qu'ils traversent et sur un certain exotisme de cette machine (Polino, 2007). 


\section{De l'objet technique à un espace touristique}

Les locomotives et les wagons peuvent apporter une valeur supplémentaire, un attrait qui influence le choix des touristes. C'est à partir de ce moment que le train «n'est plus seulement un moyen de transport, y compris au profit d'un déplacement touristique, mais un objet» (Polino, 2007 : 28). C'est au départ de la gare de Moscou que les passagers effectuant le voyage vers l'est prennent place à bord du train de luxe, touristique, ou du train régulier. Ils se retrouvent dans un véritable hôtel roulant. En revanche, le confort et les prestations sur place varient en fonction de la catégorie de ce dernier. Le Transsibérien le plus proposé aux clients n'est pas le train de luxe, mais un train régulier, de voyageurs et de marchands. Les voyageurs de ces trains réguliers ne sont pas, pour la plupart, des touristes, mais bien des passagers russes, mongols et chinois qui rentrent chez eux, car ce moyen de transport est beaucoup plus économique que l'avion.

Chaque wagon de train longue distance sur le Transsibérien en Russie est organisé de la même manière. Il est composé de deux toilettes avec lavabo, 18 couchettes (compartiment à 2 places) ou 36 couchettes (compartiment à 4 places), un compartiment pour le steward du wagon et un samovar (eau bouillante potable). Il existe trois catégories de wagons longue distance : de la troisième (économique) à la première classe (plus d'espace). Le prix d'un billet de train pour un trajet Moscou-Vladivostok varie entre 10102 roubles (224 euros) pour la troisième classe, 16526 roubles (367 euros) pour la seconde et 31014 roubles (689 euros) pour la première. Les différences entre chaque classe sont celles de confort, d'espace, d'ouverture et de fermeture. Le wagon-restaurant se distingue des autres : il est ouvert de $10 \mathrm{~h}$ à $22 \mathrm{~h}$ (heure locale) et offre en général de la nourriture préparée sur place. À chaque pays, il s'adapte aux cuisines locales (Chemins de fer russes, 2013).

Les trains de luxe, le Grand Transsibérien Express et l'Or des Tsars Zarengold, vont de Moscou jusqu'à Pékin, tandis que le Golden Eagle relie Moscou à Vladivostok. Ces voyages sont souvent organisés pendant une période de temps limité allant du début mai à la fin septembre et sont destinés principalement à la clientèle étrangère. Composés de 14 à 19 wagons passagers (avec une capacité d'accueil d'entre 120 et 150 passagers), ces trains comportent des cabines de taille et de confort différents en lien avec la présence ou l'absence des sanitaires et des douches au sein des ces dernières. On y trouve également les cabines de la classe luxe proposant les prestations haut de gamme (espace salon, bureau etc.) En règle générale, on dénombre entre deux et quatre wagonsrestaurants, un bar, un wagon conférence pour en apprendre plus sur la culture et la langue russe, un wagon pour le personnel et un wagon pour transporter les réserves au sein de chaque train de luxe.

\section{Un chemin de fer historique et des imaginaires}

Le Transsibérien a une longue histoire. Il parcourt la Russie d'est en ouest et suscite depuis sa construction fascination et curiosité. Il a été créé à la jonction des $\mathrm{XIX}^{\mathrm{e}}$ et $\mathrm{XX}^{\mathrm{e}}$ siècles (1891-1916) suite à la construction du Transcanadien. Ce dernier était une véritable source d'inspiration pour le gouverneur de la Sibérie, Mouraviev-Amourski. C'est sous le règne du tsar Alexandre III et surtout sous le règne de son fils le tsar Nicolas II que les travaux vont réellement commencer (Senin, 2001).

Véritable «colonne vertébrale» du développement sibérien, il a été créé principalement dans un but économique - afin de pouvoir jouir des richesses souterraines (minéraux de fer de l'Oural, gisements houillers) et de permettre une croissance rapide de certaines villes se trouvant sur le chemin de fer, mais également stratégique - afin de pouvoir gérer d'éventuelles tentatives de conquête de la Sibérie par ses voisins asiatiques, et ainsi de pouvoir s'imposer et affirmer une présence en Asie orientale. Ce projet novateur et ambitieux permettra également à la Russie de se rapprocher de villes occidentales telles que Paris ou Londres, apparaissant comme un signe de prestige (Senin, 2001).

Le chemin de fer transsibérien est omniprésent au sein de l'écriture artistique (dans le domaine de la littérature, de la chanson ou du cinéma). Le Transsibérien est un voyage mythique, une expérience humaine inédite apportant de la magie dans la vie des gens. Cette magie est vraiment spécifique : "sept jours de vie commune, dans un espace exigu et coupé du monde, sans échappatoire, tous égaux dans l'aventure» (Lauzanne, 2003). Le Transsibérien est synonyme d'aventure ou de rêve. En 1913, Blaise Cendrars (2006) a écrit sur ce voyage et sur ce train l'un des plus beaux poèmes de la langue française : «La prose du Transsibérien et de la petite Jeanne de France». La structure du poème, la longueur de ses vers miment le rythme du train et permettent aux lecteurs d'appréhender la vitesse de celui-ci selon la longueur des phrases. Chantée par les aventuriers et les poètes, cette voie mythique de chemin de fer fait toujours partie de la vie des Russes. C'est ainsi que l'on trouve très facilement des chants de l'époque soviétique et postsoviétique contant les gloires et les déboires du Transsibérien (Alexei Matiouchin, Artem Jyliakov, Vladimir Efimov, Sergei Chynkarenko et d'autres). Les films d'aventures mettant en scène un train comme lieu où se passe l'action sont assez nombreux (la production soviétique de 1977 Transsibérien, ou le thriller européen Transsiberian de Brad Anderson, 2008). Le train est représenté en tant que lieu de mystère, de suspense, avec différents phénomènes obscurs (crimes, trafic de drogue, etc.)

Tous ces éléments ont contribué à la création de cet objet mythique «Transsibérien» qui continue de nourrir l'imaginaire et le rêve des touristes potentiels.

\section{Le Transsibérien au sein des produits touristiques}

Le train n'est pas seulement un moyen de transport, c'est un lieu touristique. En cela, il se rapproche des autocars et des croisières : circuits en autocar, croisières ferroviaires, fluviales et maritimes. Comme pour le train, des entreprises ont fait des navires un réel segment de marché (Dehoorne et al., 2009). Le parallèle entre les croisières fluviales ou maritimes et les croisières ferroviaires est possible. Le train est un objet du quotidien. Mais souvent il suscite également le rêve, par sa dimension patrimoniale et même affective, ce qui détermine le potentiel important du marché des trains. En effet, et cela encore plus que pour la croisière maritime, les croisières ferroviaires doivent leur renommée au train dont elles portent 
le nom : Transsibérien, Orient Express, Transcanadien. Ce fait leur permet de continuer à faire partie des éléments des produits touristiques et d'avoir leur public. Ainsi, on compte plusieurs spécialistes du produit sur les marchés, sans compter les spécialistes de chacune des destinations le proposant dans sa production.

À partir de la recension des voyagistes de croisières ferroviaires du magazine Echo touristique (Gomez, 2010), j'ai étudié les offres de 9 voyagistes: Groupe Nouvelles Frontières, Amslav Tourisme, Salaün Holidays/Pouchkine Tours, Intermèdes, Voyageurs du Monde, Kuoni (Émotions) (2011), le spécialiste monégasque du train QCNS Cruise \& Trains, l'association Slaventures ainsi que le spécialiste suisse du Transsibérien Espace Est-Ouest (un exemple de l'analyse des produits «Transsibérien » sera présenté dans le point 2.1 de cet article dans le tableau 1). L'analyse des catalogues des voyagistes a démontré que les tarifs pratiqués varient entre 1150 euros (version économique) et 4800 euros pour le circuit ordinaire contre 5499 et 14450 euros pour un produit de luxe. Selon mes calculs, l'offre de luxe est moins proposée $(21,4 \%)$ que l'offre du train ordinaire (78,6\% des 30 produits étudiés). La plupart des voyagistes incluent les vols aériens internationaux ainsi que les transferts et transports intérieurs dans leur prix. L'hébergement est inclus dans tous les circuits et peut se faire en hôtel 3 ou 4 étoiles, en chalet, en maison d'hôte (à Irkoutsk ou au village typique sibérien Listvianka), sous une yourte en Mongolie (Dugana) ou bien à bord du train (en fonction de la catégorie de cabine choisie). La pension complète est proposée de façon quasi systématique pour le train de luxe. La plupart des autres circuits offrent le petit-déjeuner et les dîners à l'hôtel. Les repas à bord du Transsibérien sont rarement inclus dans l'offre traditionnelle.

Pour l'accompagnement des touristes, chaque voyagiste prévoit un guide francophone (ou anglophone) ou bien un guide local pour les suivre à chaque étape de leur circuit. Certains voyagistes proposent des services supplémentaires attractifs qui complètent les prestations générales afin de se différencier des concurrents. Il s'agit de circuits haut de gamme. Ainsi, l'association Slaventures, spécialisée dans l'organisation des voyages en Russie, propose un spectacle traditionnel et une randonnée équestre ou pédestre pendant le circuit (Slaventures, 2011). Le voyagiste Amslav, quant à lui, offre une croisière en bateau sur le lac Baïkal suivie d'un déjeuner et dîner pique-nique au bord du lac, un dîner d'adieu de canard laqué à Pékin, un programme d'animation à bord (comprenant des conférences sur l'histoire et la géographie des régions et des pays traversés), le port des bagages dans toutes les gares ainsi qu'une dégustation de caviar et de vodka dans tous les circuits proposés. Ce voyagiste met en valeur la possibilité pour les touristes de passer sur l'ancien tracé du Transsibérien le long du lac Baïkal (autorisé uniquement pour le train l'Or des Tsars) (Amslav, 2011). En général, le prix du circuit ne comprend pas l'assistance rapatriement, l'assurance annulation, les frais de visa, les dépenses et les frais personnels ainsi que les visites optionnelles et les pourboires. En revanche, les prestations liées aux démarches administratives sont proposées par les professionnels à condition de payer un supplément.

\section{Les lieux touristiques selon I'analyse fréquentielle de leurs fonctions}

Ayant présenté la variété de l'offre de produits organisés autour du train, nous allons nous pencher à présent sur l'investissement, par les trains, des lieux traversés, et de comment ils affectent (ou non) le développement touristique de ces derniers. L'étude de ce «degré» de dépendance du territoire du passage du train sera effectuée sous le prisme de l'analyse du développement territorial. Pour cela, nous utilisons la méthodologie permettant de produire des données plus fiables que les statistiques officielles sur les lieux-étapes et les lieux visités par les touristes.

\section{La méthodologie adaptée qui permet de mettre en lumière le lien entre le train et le tourisme} La méthode de production de données proposée par Philippe Violier (2011) repose sur l'analyse des catalogues des voyagistes (il s'agit dans notre cas des professionnels français proposant le Transsibérien) et consiste à effectuer une «analyse fréquentielle des lieux nommés dans les circuits (mode de déplacement touristique qui consiste à recourir à plusieurs lieux d'hébergements successifs) et séjours (un lieu unique d'hébergement), et des fonctions de ces lieux en relation avec la typologie des lieux touristiques élaborée également par l'Équipe Miт (2002; Stock, 2003). Cette dernière distingue les lieux créés (comptoirs et stations) des lieux investis (site et villes touristiques)»(Équipe Mrt, 2002). L'analyse fréquentielle est bien adaptée à l'étude des interactions entre tourisme et transport, car elle permet, d'une part, d'identifier l'importance des lieux du point de vue touristique sur les différents parcours des trains. D'autre part, cette méthode permet de produire des données fiables concernant le Transsibérien, face à la complexité du recueil des statistiques produites par les cadres politiques administratifs des trois pays concernés (la Russie, le Mongolie et la Chine). Les textes des catalogues sont plus ou moins développés. D’une manière générale, le volume d'information présent dans un catalogue est en relation directe avec le positionnement économique.

Pour sélectionner les voyagistes, j'ai utilisé le palmarès des voyagistes français sur la destination Russie établie par le magazine Echo touristique (Gomez, 2010). J'ai choisi les 5 voyagistes de cette liste (Groupe Nouvelles Frontières, Amslav Tourisme, Salaün Holidays/Pouchkine Tours, Intermèdes (2011), Voyageurs du Monde) qui proposent le Transsibérien, y acheminant plus de 200 touristes par an. Leurs catalogues de voyages sont diffusés soit par l'imprimé, soit via Internet. J'ai aussi choisi d'inclure le spécialiste du train QCNS Cruise \& Trains (distributeur monégasque des principales compagnies ferroviaires de luxe) à cette liste afin de pouvoir comparer ses offres avec celles des voyagistes, ainsi que l'association Slaventures et le spécialiste suisse du Transsibérien Espace EstOuest proposant 12 produits "Transsibérien », et le voyagiste Kuoni (Émotions) absent du palmarès 2010, mais présent dans le bilan de 2009. Ensuite, une fois les voyagistes sélectionnés, j'ai étudié les circuits proposés et établi un tableau par voyagiste (tableau 1). À partir de ces éléments, une analyse a été effectuée, portant d'un côté sur la fréquence des lieux 
Tableau 1: Exemple de l'analyse des 4 produits «Transsibérien » du voyagiste Salaün Holidays/Pouchkine Tours (2012)

\begin{tabular}{|c|c|c|c|c|c|c|}
\hline $\begin{array}{l}\text { Nom du produit } \\
\text { "Transsibérien " }\end{array}$ & $\begin{array}{l}\text { Tronçon (départ } \\
\text { de Paris) }\end{array}$ & $\begin{array}{l}\text { Nombre de nuits } \\
\text { en général et dans } \\
\text { le train }\end{array}$ & Lieux-étapes et lieux visités & Tarif & Le prix inclut & $\begin{array}{c}\text { Le prix } \\
\text { n'inclut pas }\end{array}$ \\
\hline $\begin{array}{l}\text { Voyage dans l'express } \\
\text { Europe-Asie }\end{array}$ & Moscou - Pékin & $\begin{array}{l}16 \text { jours / } 15 \text { nuits } \\
\text { dont } 6 \text { nuits dans } \\
\text { le train }\end{array}$ & $\begin{array}{l}\text { * Moscou (avion) - } 3 \text { nuitées } \\
\text { * Serguiev Possad } \\
\text { * Oulan-Bator - } 1 \text { nuitée } \\
\text { (Transmongolien) } \\
\text { * Dugana - } 2 \text { nuitées } \\
\text { (nuit sous une yourte } \\
\text { traditionnelle } \\
\text { * Pékin - } 3 \text { nuitées }\end{array}$ & $2775 €$ & $\begin{array}{l}\text { * Le transport aérien }+ \text { taxes } \\
\text { * Nuits dans le train Moscou - } \\
\text { Oulan-Bator en } 1^{\text {re }} \text { ou } 2^{\text {de }} \text {, } \\
\text { et en } 2^{\text {de }} \text { sur le trajet } \\
\text { Oulan-Bator - Pékin } \\
\text { * L'hébergement en hôtel } \\
\text { * La pension complète } \\
\text { * Les transferts } \\
\text { * Le transport } \\
\text { * Les visites et excursions + } \\
\text { guide francophone } \\
\text { * Assurance rapatriement }\end{array}$ & $\begin{array}{l}\text { * Tous les } \\
\text { repas à } \\
\text { bord du } \\
\text { Transsibérien } \\
\text { *Visa } \\
{ }^{*} \text { Pourboire }\end{array}$ \\
\hline $\begin{array}{l}\text { Voyage dans le train de } \\
\text { tous les records }\end{array}$ & Moscou - Pékin & $\begin{array}{l}19 \text { jours / } 18 \text { nuits } \\
\text { dont } 7 \text { nuits dans } \\
\text { le train }\end{array}$ & $\begin{array}{l}\text { * Moscou (avion) }-3 \text { nuitées } \\
\text { * Ekaterinbourg }-1 \text { nuitée } \\
\text { * Irkoutsk } \\
\text { * Listvianka }-1 \text { nuitée } \\
\text { * Oust-Orda } \\
\text { * Oulan-Bator }-2 \text { nuitées } \\
\text { (Transmongolien) } \\
\text { * Dugana }-1 \text { nuitée (nuit sous } \\
\text { yourte) } \\
\text { * Pékin }-3 \text { nuitées }\end{array}$ & $3775 €$ & $\begin{array}{l}\text { * Le transport aérien }+ \text { taxes } \\
{ }^{*} \text { Nuits dans le train en } 1^{\text {re }} \text { ou } 2^{\text {de }} \\
\text { * L'hébergement en hôtel } \\
{ }^{*} \text { La pension complète } \\
\text { * Les transferts } \\
{ }^{*} \text { Le transport } \\
\text { * Les visites et excursions }+ \\
\text { guide francophone } \\
\text { * Assurance rapatriement }\end{array}$ & $\begin{array}{l}\text { * Tous les } \\
\text { repas à } \\
\text { bord du } \\
\text { Transsibérien } \\
\text { *Visa } \\
\text { * Pourboire }\end{array}$ \\
\hline $\begin{array}{l}\text { Voyage dans le train } \\
\text { des steppes }\end{array}$ & Irkoutsk - Pékin & $\begin{array}{l}15 \text { jours / } 14 \text { nuits } \\
\text { dont } 3 \text { nuits dans le } \\
\text { train }+1 \text { nuit dans } \\
\text { l'avion (Moscou - } \\
\text { Irkoutsk) }\end{array}$ & $\begin{array}{l}\text { * Moscou (avion) - } 2 \text { nuitées } \\
{ }^{*} \text { Serguiev Possad } \\
\text { * Irkoutsk - } 3 \text { nuitées } \\
\text { * Lac Baïkal + Listvianka } \\
\text { * Oust-Orda } \\
{ }^{*} \text { Oulan-Oudé } \\
\text { * Oulan-Bator - } 1 \text { nuitée } \\
\text { * Désert de Gobi } \\
\text { * Dugana - } 1 \text { nuitée } \\
\text { * Pékin - } 3 \text { nuitées }\end{array}$ & $3195 €$ & $\begin{array}{l}\text { * Le transport aérien + taxes } \\
{ }^{*} \text { Nuits dans le train en } 2^{\mathrm{de}} \\
\text { Attention : si le train est } \\
\text { réquisitionné par les autorités } \\
\text { mongoles, le trajet se fera en } \\
\text { avion (pour la partie Mongolie) } \\
\text { * L'hébergement en hôtel } \\
\text { * La pension complète } \\
\text { * Les transferts } \\
\text { * Le transport } \\
\text { * Les visites et excursions + } \\
\text { guide francophone } \\
\text { * Assurance rapatriement }\end{array}$ & $\begin{array}{l}{ }^{*} \text { Assurances } \\
{ }^{*} \text { Les repas } \\
\text { à bord du } \\
\text { train (demi- } \\
\text { pension en } \\
\text { supplément), } \\
\quad{ }^{*} \text { Visa }\end{array}$ \\
\hline $\begin{array}{l}\text { Collection prestige (le } \\
\text { train de luxe) }\end{array}$ & Moscou - Pékin & $\begin{array}{l}16 \text { jours / } 15 \text { nuits } \\
\text { dont } 9 \text { nuits dans } \\
\text { le train }\end{array}$ & 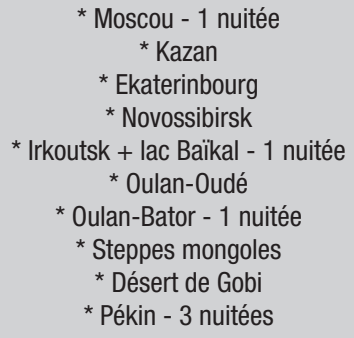 & $6395 €$ & $\begin{array}{l}\text { * Le transport aérien }+ \text { taxes } \\
\text { * Nuits dans le train en } 1^{\mathrm{re}} \text { ou } 2^{\mathrm{de}} \\
{ }^{*} \text { L'hébergement en hôtel } \\
{ }^{*} \text { La pension complète } \\
{ }^{*} \text { Les visites et excursions }+ \\
\text { guide francophone } \\
{ }^{*} \text { Assurance rapatriement }\end{array}$ & $\begin{array}{c}\text { * Les boissons } \\
\text { au cours des } \\
\text { repas (autres } \\
\text { que celles } \\
\text { mentionnées) } \\
\text { *Visa }\end{array}$ \\
\hline
\end{tabular}

Source : compilation de l'auteure.

cités en tant qu'étape ou lieu visité, et, d'un autre côté, sur les prestations proposées à bord du train en fonction de l'offre (luxueuse ou traditionnelle). Le calcul du nombre de nuits passées à bord du train nous permet de quantifier l'importance du train dans l'offre touristique.

Le tableau 1 départage les lieux-étapes (nuitées) des lieux visités par les touristes passagers du Transsibérien afin de pouvoir établir la hiérarchie des lieux. Je me suis principalement intéressée aux lieux de concentration de différentes pratiques des touristes.
L'analyse fréquentielle employée ici a permis d'établir deux cartes des lieux fréquentés sur le parcours du train transsibérien. La première reflète le nombre des nuitées (illustration 2) tandis que la deuxième présente les lieux visités dans lesquels les touristes ne passent pas de nuit (illustration 3).

Selon mes calculs, les Transsibériens (trains ordinaires et de luxe) arrivent en tête en nombres de nuitées par rapport à des villes-étapes figurant dans les catalogues. Ils comptabilisent près de $50 \%$ de la totalité des nuitées effectuées dans le cadre des circuits étudiés. Le nombre de nuitées passées à bord 


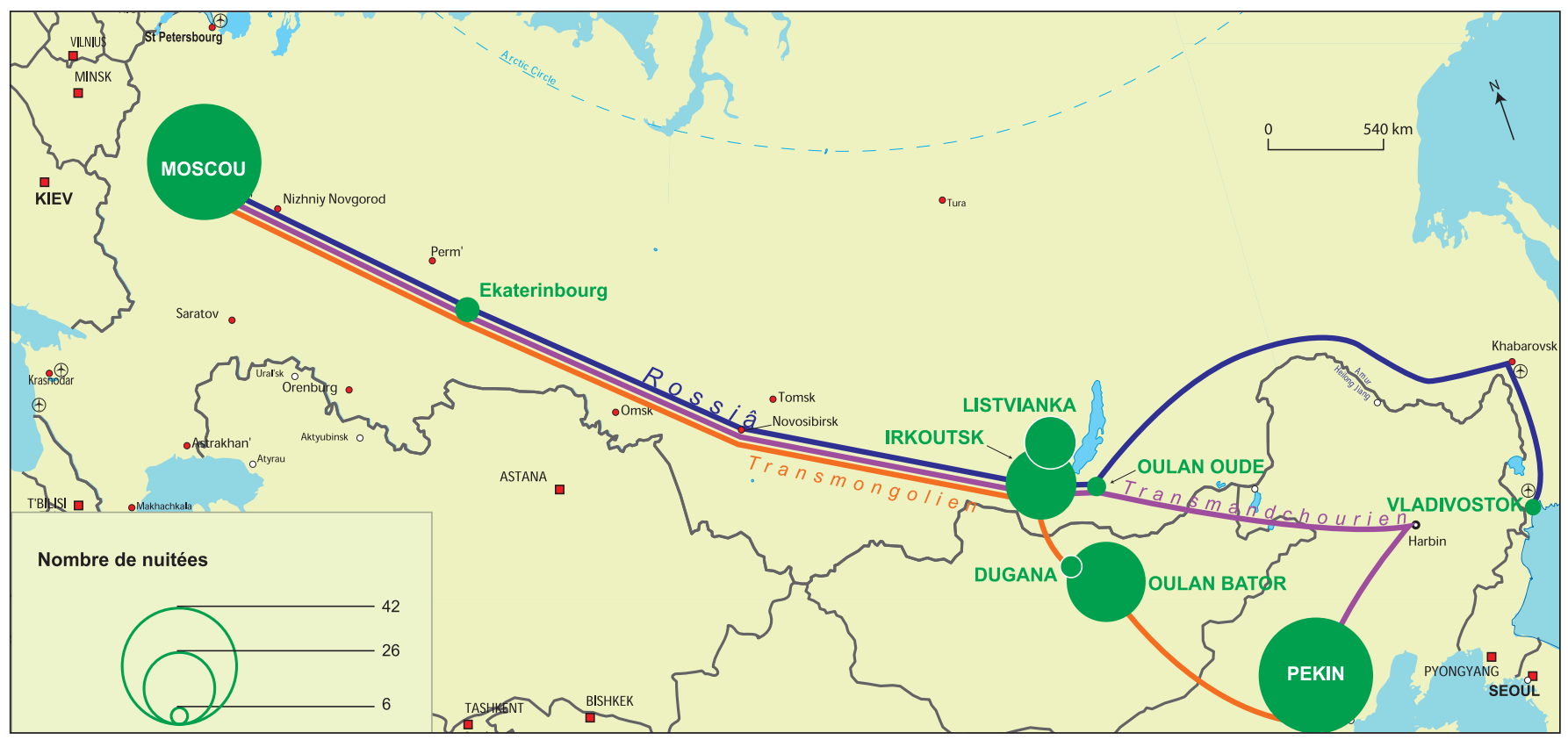

ILLUSTRATION 2 : Fréquence des lieux-étapes cités (hors nuits passées dans le train) dans les catalogues des voyagistes et des spécialistes du train étudiés (source : conception de l'auteure).

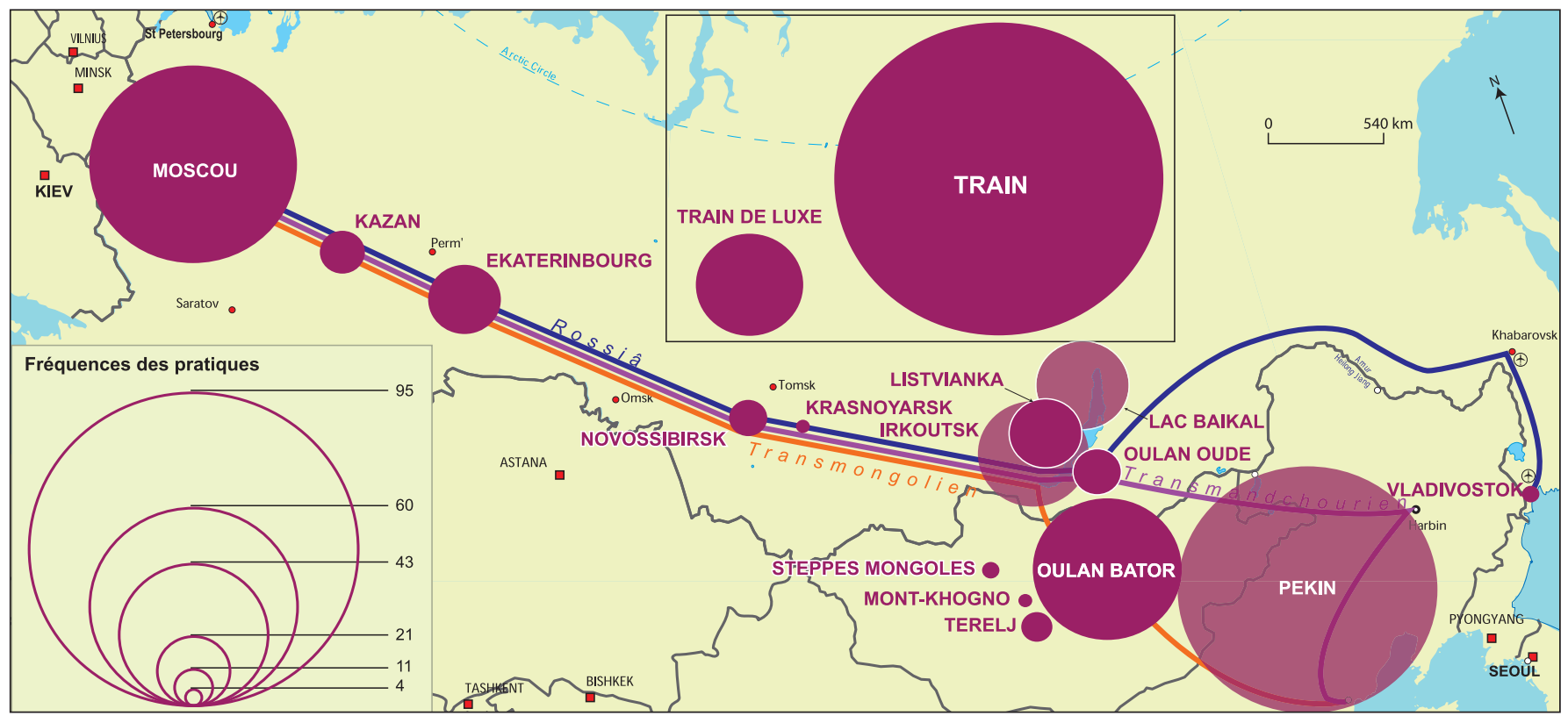

ILLUSTRATION 3 : Fréquence des lieux visités cités dans les catalogues de voyagistes et des spécialistes du train étudiés (source : conception de l'auteure).

des trains ordinaires s'élève à 26,27\% sur la totalité de nuitées. Le train de luxe arrive avec près de $21 \%$ de nuitées. Ces chiffres illustrent l'importance du train dans cette offre touristique.

Néanmoins, 4 des 8 voyagistes étudiés proposent une «aventure» à bord du Transsibérien pour seulement 3 ou 4 nuits passées dans le train. C'est le cas de Nouvelles Frontières (2011), proposant «un voyage d'exception à bord du mythique Transsibérien» avec les 3 nuits passées dans le train sur le trajet reliant Irkoutsk à Pékin, ou de Salaün Holidays et son produit «Voyage dans le train des steppes» proposant le trajet Moscou-Pékin avec la liaison aérienne Moscou-Irkoutsk et 3 nuits à bord du train (Salaün Holidays, 2012 : 125). Voyageurs du Monde, dans son offre Moscou-Irkoutsk, propose à ses clients «une immersion dans la Russie profonde à bord d'un train mythique» pour 4 nuits passées dans le train (Voyageurs du Monde, 2011). Un autre exemple est le produit d'Amslav intitulé "Transsibérien de Moscou à Irkoutsk», qui comprend seulement 4 nuits à bord (Amslav, 2011). Ce fait peut 
s'expliquer d'un côté par la durée de ces deux Transsibériens qui, contrairement aux autres, ne font que la moitié du parcours (au départ et à destination d'Irkoutsk). D’un autre côté, les nuits en train se font en un seul trajet, sans escale, ce qui montre l'importance du train dans cette offre touristique proposée par les voyagistes.

Les capitales des trois pays traversés par le train, les villes de Moscou, Oulan-Bator et Pékin, comptabilisent un grand nombre de nuitées. Cela s'explique par la notoriété de ces lieux et par une offre touristique importante, ce qui rallonge les séjours des touristes sur place. Un deuxième pôle de concentration d'un grand nombre de nuitées est représenté par la région d'Irkoutsk et lac Baïkal (le plus ancien et le plus profond lac du monde). Les voyagistes ont l'habitude de proposer l'hébergement à Irkoutsk ou au village Listvianka, situé à 66 kilomètres de la ville.

L'étude approfondie des catalogues des voyagistes, étude dont les résultats sont présentés sous forme de carte (illustration 3), permet de constater que l'offre nommée «Transsibérien» et proposée par différents voyagistes français est caractérisée par un grand nombre de similitudes. L'illustration 3 montre de façon évidente l'existence des points de concentration des lieux sur le trajet du Transsibérien associés aux différentes pratiques touristiques. En effet, le Transsibérien comme itinéraire touristique peut avoir plusieurs variations en fonction du parcours choisi, mais les villes de Moscou (avec 15,26\% de fréquences enregistrées), de Kazan $(3,3 \%)$, d'Ekaterinbourg $(5,34 \%)$, de Novossibirsk $(2,8 \%)$ et d'Irkoutsk $(8,14 \%)$ avec le lac Baïkal $(6,87 \%)$ resteront ses étapes principales. Je ne prends pas en compte les pratiques à bord des trains pour le calcul de ces pourcentages, car je ne m’intéresse ici qu'aux lieux traversés par le train.

En revanche, c'est l'offre du train Transmongolien invitant les touristes à découvrir les trois pays différents qui est la plus proposée par les voyagistes. Elle représente $55 \%$ de l'ensemble des offres (le calcul est basé sur les 30 circuits étudiés). Par conséquent, on ne peut pas nier l'importance de la place occupée par la capitale chinoise comme principal lieu de concentration des pratiques touristiques (avec 19\% des fréquences obtenues), ainsi que la capitale de la Mongolie, Oulan-Bator, qui compte un peu plus de $10 \%$ de fréquences. Les escales plus atypiques sont proposées par certains voyagistes, principalement en Mongolie. C'est le cas des villes-étapes comme Terelj (nuit sous une yourte traditionnelle proposée par Nouvelles Frontières et Amslav), Hustai (Nouvelles Frontières), Sainshand (Slaventures).

La méthodologie utilisée lors de ce travail de recherche possède des limites. Premièrement, les catalogues des voyagistes représentent une sorte de suggestion de voyages, adressée aux clients potentiels. On ne peut donc pas avoir une certitude sur la réalisation de ces circuits. En revanche, compte tenu du coût de l'édition de ces catalogues, les voyagistes y conservent les circuits les plus vendus (Violier, 2011).

Deuxièmement, on peut contester le fait que cette méthode ne permette pas d'inclure dans les statistiques les personnes qui organisent leur voyage de manière individuelle, sans passer par un intermédiaire. Selon l'enquête "Vacances» réalisée par l'INSEE en 2004, 48\% de personnes résidant en France s'adressent à un voyagiste pour organiser leur déplacement en dehors de la France métropolitaine (Violier, 2011). La fréquence du recours au voyagiste augmente avec l'altérité (Mondou et Violier, 2009; Violier et al., 2009; Duhamel et Violier, 2009). Dans notre cas, le voyage à bord du Transsibérien représente pour un touriste français une situation de forte altérité.

La méthodologie d'analyse des catalogues des voyagistes peut être transférée à d'autres contextes de transport, notamment dans le domaine de croisières fluviales ou maritimes. Cette méthode peut s'avérer intéressante dans le recueil de données concernant les pratiques des touristes de nationalités différentes sur place lors des escales compte tenu du fait que les croisiéristes passent la majorité de leurs nuits à bord du navire. Elle peut également permettre de mesurer l'impact produit par le passage des navires de croisières sur le développement de l'économie touristique des villes-escales.

La méthodologie utilisée pour ce travail de recherche, complétée par des recherches documentaires sur les lieux traversés par le train, permet d'identifier deux territoires pertinents à analyser en tant qu'études de cas. Ils se distinguent des autres lieux par leur forte dépendance du train. Le premier est l'oblast de Novossibirsk, dont le développement touristique a débuté avec l'arrivée du Transsibérien. Le deuxième cas est celui de Listvianka, un village typique de Sibérie situé proche $\mathrm{du}$ lac Baïkal. Tous les deux tirent bénéfice du passage du Transsibérien.

\section{Étude de cas : interaction entre le train et les territoires traversés}

Si le chemin de fer fait naitre certains lieux, il peut aussi contribuer au développement économique local, ou relancer les secteurs en difficulté en donnant un nouveau souffle à ces derniers. Nous le verrons à travers deux exemples.

\section{Un lieu né grâce au Transsibérien : Novossibirsk.}

À $2814 \mathrm{~km}$ à l'est de Moscou, Novossibirsk est la première ville de Sibérie. Elle est le centre du district fédéral (entité territoriale administrée par un plénipotentiaire nommé par le président de la fédération) de la Sibérie depuis 2000. Avec une population de 1523801 habitants en 2013, elle est la troisième ville russe après la capitale, Moscou, et la ville de Saint-Pétersbourg (Goskomstat, 2010). La ville de Novossibirsk doit sa naissance au chemin de fer transsibérien, car elle a été fondée en 1893 près du pont de ce chemin de fer pour le passage du train. La gare ferroviaire «Novossibirsk Glavniy» (gare centrale de Novossibirsk), l'un des lieux les plus visités de la ville, est la plus grande gare sur le passage du Transsibérien (Chemins de fer russes, 2013). Le territoire de l'oblast de Novossibirsk est traversé par tous les trains "Transsibérien», ce qui en fait un noud de transport important. La ville de Novossibirsk est donc l'un des arrêts principaux des parcours.

Les touristes étrangers arrivent dans la ville essentiellement par la voie ferrée, car les liaisons aériennes internationales ne sont pas populaires parmi cette clientèle. Près d'une vingtaine d'agences de voyages locales (sur 250 enregistrées) assurent l'accueil des touristes étrangers. Selon les données officielles du Gouvernement de la région de Novossibirsk (2013), le 
Tableau 2: L'évolution du nombre de touristes domestiques et étrangers à Novossibirsk de 2010 à 2013

\begin{tabular}{|c|c|c|c|c|}
\hline Année & 2010 & 2011 & 2012 & 2013 \\
\hline Nombre de touristes domestiques & 11800 & 12350 & 12850 & 13400 \\
\hline Évolution en \% par rapport à l'année précédente & & $+4,7 \%$ & $+4 \%$ & $+4,2 \%$ \\
\hline Nombre de touristes étrangers & 65700 & 67300 & 70500 & 74000 \\
\hline Évolution en \% par rapport à l'année précédente & & $+2,4 \%$ & $+4,75 \%$ & $+5 \%$ \\
\hline
\end{tabular}

nombre des touristes étrangers augmente depuis quelques années de manière considérable (tableau 2).

Les touristes domestiques sont moins nombreux par rapport aux étrangers, on compte près de 6 touristes internationaux pour un touriste russe à Novossibirsk. Malgré une augmentation du nombre de touristes domestiques à Novossibirsk d'une année à l'autre, le tableau 1 montre une évolution plus importante du côté des touristes étrangers. Les statistiques produites par l'État russe en matière de tourisme sont toutefois biaisées. Une surévaluation des chiffres peut être entretenue de façon volontaire par l'État avec l'objectif de rassurer et d'attirer les investisseurs étrangers, ou pour donner une bonne image des politiques menées par le gouvernement (Andreeva - Jourdain, 2014).

L'analyse des catalogues des voyagistes français montre que, en général, les touristes étrangers font une escale de 1 ou 2 jours à Novossibirsk. Depuis quelques années, la ville trouve de plus en plus d'intérêt aux yeux des touristes étrangers grâce à son dynamisme et à son offre large de divertissements (restaurants, bars, etc.) Les clients peuvent également bénéficier d'un moment de repos après le voyage en train et ont la possibilité de découvrir la vie d'un grand centre scientifique et culturel de la Sibérie, ce qui motive les voyagistes étrangers à y proposer des escales plus conséquentes. Le secteur hôtelier étant le premier à être concerné par ces changements, il a dû s'adapter. En 2011, la ville de Novossibirsk comptait 124 hôtels avec une capacité d'accueil de 7500 personnes par jour (Bourdastova, 2013 : 142). Nous sommes loin de la saturation, car la ville compte seulement 3 places dans le secteur hôtelier marchand pour 1000 habitants. Les hébergements du «type soviétique» - les complexes hôteliers en chandelle avec une grande capacité d'accueil (hôtel Novossibirsk situé près de la gare, hôtel Central, Azimut Hotel Sibir (ancien hôtel Sibérie), River Park (ancien hôtel $\mathrm{Ob}$ ) - commencent à être concurrencés par les chaînes hôtelières internationales. Depuis 5 ans, 24 nouveaux hôtels ont ouvert leurs portes. Ainsi, Hilton a inauguré en octobre 2010 son établissement Doubletree by Hilton Novossibirsk (Bourdastova, 2013 : 142). Il s'agit du premier hôtel de la ville ouvert sous une marque internationale. Le Doubletree comporte 188 chambres et deux restaurants, un spa, un bar et une salle de sport, le tout à quelques minutes à pied du centre-ville. Les concurrents de Hilton, les groupes InterContinental, Accor et Swissôtel, ont tous prévu d'ouvrir leurs propres hôtels en ville. Le premier hôtel de la catégorie 5 étoiles a été dirigé par l'entrepreneur hôtelier international Marriott Hotels
\& Resorts. L'hôtel de 175 chambres, situé devant le Théâtre d'opéra et de ballet, en centre-ville, a été ouvert en juin 2014. Le marché du secteur d'hébergement de l'oblast de Novossibirsk a connu une augmentation de $40 \%$ en 2012 par rapport à l'année 2011 (Bourdastova, 2013 : 142). Les acteurs publics locaux ont également compris l'importance croissante de cette clientèle internationale en termes de retombées économiques pour les territoires d'accueil. L’oblast de Novossibirsk bénéficie d'un programme nommé «Le développement du tourisme pendant la période 2012-2016», élaboré selon la Stratégie de développement socio-économique de l'oblast allant jusqu'à 2025 et approuvé par le gouverneur de l'oblast le 3 décembre 2007 dans l'arrêté numéro 474 (Gouvernement de la région de Novossibirsk, 2007). Ce programme prévoit la mise en place des mesures visant entre autres la définition du niveau de soutien étatique de l'industrie touristique ainsi que l'implication des acteurs du secteur privé dans le développement de l'infrastructure touristique de l'oblast. Des accents particuliers sont placés par le pouvoir local sur le développement des transports et de la qualité des prestations proposées sur place. Il s'agit donc de la mise en place d'une stratégie commune de développement économique à travers le tourisme grâce à laquelle les autorités locales envisagent de recevoir 75000 touristes étrangers en 2014, 76000 en 2015 et 77000 en 2016 (Gouvernement de la région de Novossibirsk, $2011: 18$ ).

\section{Les lieux qui ont trouvé leur nouveau souffle grâce au Transsibérien : I'exemple de Listvianka}

Le village Listvennichnoe (connu sous le nom de Listvianka) longe la rive occidentale du lac Baïkal sur plusieurs kilomètres et compte 1834 habitants (Goskomstat, 2010). Son cas est intéressant, car il illustre comment un lieu développé grâce au train, et coupé d'accès à ce dernier par la suite, a su s'adapter à ces changements pour redevenir à nouveau lié au train dans le cadre d'une stratégie étatique de développement touristique. Cet exemple montre donc un autre type d'interaction possible entre le train et le territoire. Au début du XVIII ${ }^{e}$ siècle, le train n'existait pas au moment de la fondation du village, qui s'est illustré par la construction des bateaux dans ses chantiers navals et par la pêche, deux activités principales tout le long des XIX ${ }^{\mathrm{e}}$ et $\mathrm{XX}^{\mathrm{e}}$ siècles. Depuis les années 1920, une station limnologique (science des eaux superficielles continentales ou intérieures) a été créée au village, ce qui a attiré un grand nombre de scientifiques. L'histoire du village a été longtemps liée à celle du chemin de fer transsibérien, qui a joué un rôle 
important dans le développement économique de ce dernier (Goldfarb, 2002).

Jusqu'en 1956, l'accès au village s'effectuait par la voie ferrée de l'ancien Transsibérien. En 1957, après la construction du barrage de la centrale hydroélectrique d'Irkoutsk, un tronçon de la voie ferrée (d'Irkoutsk jusqu'au lac Baïkal le long de l'Angara) a été inondé. Cette partie de la voie ferrée a été reconstruite dans les montagnes en contournant le lac. À la fin des années 1970, cette partie du Transsibérien, devenue une impasse, a reçu le statut de monument architectural et historique, et a été mise sous la protection de l'État.

Le développement de Listvianka a connu des changements importants au début des années 1990 avec l'arrêt des subventions étatiques destinées aux projets scientifiques et la mise à l'abandon des chantiers navals. Grâce à l'ouverture de la Russie au tourisme international après la chute de l'URSS en 1991 et à sa position en avant-scène du lac Baïkal, les autorités régionales et les entrepreneurs du village ont alors commencé à miser sur le tourisme. Les maisons d'hôtes, les restaurants, les points de location de bateaux tenus par les habitants du village vont apparaître rapidement pendant cette période. La plupart des touristes arrivant dans la région du lac Baikal commencent la découverte par ce village de pêcheurs. En tant que principal pôle touristique du lac, le village de Listvianka est visité par environ 300000 touristes par an dont un quart (75 000) sont des touristes étrangers (IrkutskMedia, 2012).

Aujourd'hui, l'ancien tronçon de 84 kilomètres, représentant les exemples de l'ingénierie architecturale ferroviaire, fait partie des lieux visités par les touristes. Plusieurs projets touristiques mis en place par les pouvoirs locaux visent une patrimonialisation ferroviaire de cette partie du chemin de fer. Le village de Listvianka et le chemin de fer circumbaïkalien sont inclus dans la stratégie du développement spatial mise en place par la municipalité du Port Baïkal (village situé en face de Listvianka), qui prévoit l'élaboration de programmes de développement du transport ferroviaire touristique sur l'ancien chemin de fer (IrkutskMedia, 2012).

De plus, Listvianka ainsi que le chemin de fer circumbaïkalien seront intégrés a posteriori dans un pôle de compétitivité dans le domaine du tourisme de la Zone Économique Spéciale (ZES) «l'Anneau de Baikkal» (Kommersant, 2013) créé en 2007. Il s'agit d'un mécanisme ayant pour objectif d'accélérer le développement du tourisme sur ses hauts lieux à l'échelle du pays. L'État accorde des privilèges exclusifs aux entreprises spécialisées dans le secteur du tourisme implantées sur ces territoires (Loi fédérale, 2005). Ce projet, dont les débuts datent de l'année 2007, a déjà contribué de façon indirecte au développement du tourisme au village dans les années 2007-2008. C'est à cette période que Listvianka a connu une nouvelle vague dans le développement des initiatives privées dans le secteur du tourisme. En effet, le point de départ pour ces initiatives était le projet de soutien étatique nommé «Baikal city». Ce dernier s'inscrivait dans le processus de création des ZES choisies à la suite d'un concours fédéral pour le tourisme et les loisirs datant de février 2007. Selon ce projet, le village de Listvianka devait devenir un important centre des voyages d'affaires au niveau international, pouvant accueillir un million de touristes par an. Finalement, l'État a choisi le village de Bolchoyé Goloustnoyé en tant que pivot de ce projet, renonçant par ce fait au développement du tourisme à Listvianka de manière centralisée. Néanmoins, les entrepreneurs locaux vont profiter d'une certaine publicité produite par le projet pour faire développer les infrastructures du lieu (Kommersant, 2013). Ainsi, en 2007, le nombre d'entreprises d'hébergement (tous types confondus) appartenant au secteur marchand s'élevait à 87 (Alekseeva, 2007 : 2). Différents complexes touristiques et villas ont été construits récemment ou sont en cours de construction. Les cafés, les restaurants, le marché de poisson ont été spécialement conçus pour les touristes. Le quai abrite les points de location de bateaux de promenade sur le lac Baïkal.

Désormais, c'est l'État qui souhaite profiter du développement touristique des lieux comme Listvianka en l'incluant dans cette ZES et en lui confiant le statut de territoire-modèle de la région d'Irkoutsk. Ce processus va contribuer au développement des territoires voisins (les villages Port Baikal, Nicolas, Bol'shie Koti). Le soutien financier de l'État dans le cadre de ce projet qui va jusqu'en 2026 s'élève à 55 millions d'euros contre 155 millions d'euros d'investissements privés attendus (Kommersant, 2013). On voit grâce à ces exemples l'apparition des nouvelles logiques dans le développement touristique du village et de ses alentours.

\section{Conclusion}

D’une manière générale, les liens entre le transport et le tourisme sont multiples. Il n'est pas rare d'associer l'émergence des nouveaux lieux touristiques ou l'augmentation de la fréquentation d'une station aux transports. Certes, le transport incite le développement du tourisme sur un territoire grâce à sa fonction principale qui est celle de donner accès tout en diffusant la mobilité. Néanmoins, le maillon transport-tourisme possède des caractéristiques plus complexes. La question de l'accessibilité des lieux dans le processus d'une mise en tourisme ne peut pas s'inscrire uniquement dans le champ des transports. On ne peut pas ignorer l'importance d'autres acteurs majeurs du développement touristique (les touristes eux-mêmes, l'hébergement touristique, les services, etc.) qui font fonctionner le système touristique.

Cet article a permis de mettre en valeur le rapport réel et explicite qui existe entre le train et le territoire à l'échelle de la Russie en prenant en compte les spécificités du pays. Nous avons surtout mis en lumière le rôle du Transsibérien dans l'apparition des nouvelles logiques touristiques visant le développement du tourisme international et intérieur en Russie à travers deux exemples évoqués. Les cas de Novossibirsk, ville née grâce au train, et de Listvianka, historiquement et économiquement un port de pêche, nous montrent comment les lieux, suite au développement des initiatives privées, se retrouvent au centre des stratégies des acteurs étatiques se positionnant de plus en plus dans le champ du tourisme.

La démarche méthodologique mise en place pour cet article montre des résultats intéressants permettant d'identifier les lieux touristiques importants sur les différents parcours des trains tout en produisant des données fiables concernant le Transsibérien. Néanmoins, elle est pertinente dans un contexte d'altérite forte. Cela serait donc judicieux pour 
la suite de cette étude de poursuivre nos investigations, d'une part, sur l'identification des dynamiques dans la répartition des nuitées en rapport avec des évolutions possibles dans le contexte régional. D'autre part, une analyse des stratégies et des concurrences d'autres voyagistes francophones ainsi que des voyagistes anglophones nous permettra d'approfondir nos recherches sur les liens entre ces stratégies et la qualité des lieux traversés par le train.

Cette analyse a mis en avant le lien fort qui existe entre les recompositions territoriales et politiques en Russie postsoviétique. Souvent, les lieux touristiques constituent un laboratoire d'observation de tendances plus générales permettant de comprendre les mutations au sein du pays. La thématique abordée dans cet article pourra être analysée dans le futur sous le prisme de la politique, dominante dans ce pays et sans laquelle les systèmes touristiques de ce dernier ne peuvent pas être compris.

\section{Références}

ALEKSEEVA, Irina (2007) «Govorim Listvianka podrazumevaem Baikal»

[On dit Listvianka en nous imaginant le Baïkal], Irkipedia, $<$ http://irkipedia.ru/content/my_govorim_listvyanka_podrazumevaem_baykal>, consulté le 7 septembre 2013.

AMSLAV (2011) Europe Centrale, Russie, Asie Centrale, catalogue 2011-2012, Uniprint. $138 \mathrm{p}$.

ANDERSON, Brad (2008) Transsibérien, film produit par Brad ANDERSON, studio First Look International, Royaume-Uni/Allemagne/Espagne.

ANDREEVA-JOURDAIN, Ekaterina (2014) «Le tourisme domestique en Russie : une affaire d'État. Approche historico-géographique à partir de l'exemple de la région du Caucase du Nord", partie 4, chapitre 4, DANS Les nouvelles frontières $d u$ tourisme mondialisées, Isabelle SACAREAU; Emmanuelle PEYVEL et Benjamin TAUNAY, Paris : Armand Colin, collection Recherches.

BELLEC, Hervé (2008) Les sirènes du Transsibérien, Brest : Géorama. 276 p.

BOURDASTOVA, Julia (2013) Le marché hôtelier russe en 2013, RBK Research, Moscou : RBK. 210 p.

CENDRARS, Blaise (2006) «La prose du Transsibérien et de la petite Jeanne de France", extrait de Du monde entier au cour du monde : poésie complète, Paris : Gallimard. 79 p.

CHEMINS DE FER RUSSES (2013) «Les horaires et offres tarifaires des prestations», Chemins de fer russes, $<$ http://pass.rzd.ru/timetable/ public/ru?STRUCTURE_ID=735>, consulté le 6 septembre 2013.

DEHOORNE, Olivier; Christelle MURAT et Nathalie PETIT-CHARLES (2009) «Le tourisme de croisière dans l'espace caribéen : évolutions récentes et enjeux de développement ", Études caribéennes, $n^{\circ} 13-14$, $<$ http://etudescaribeennes.revues.org/3843>; <DOI : 10.4000/etudescaribeennes.3843>, consulté le 25 novembre 2013.

DUHAMEL, Philippe et Philippe VIOLIER (2009) Tourisme et littoral : un enjeu du Monde, Paris : Belin. 182 p.

DUNBAR, Géraldine (2006) Seule sur le Transsibérien, Mille et une vies de Moscou à Vladivostok, Paris : Transboréal. 289 p.

DURAKOV, Oleg (2007) «Transsib : budušee, nastoâšee» [Transsib : futur, présent], $R Z D, \mathrm{n}^{\circ} 5, \mathrm{p} .3-13$.

ÉQUIPE MIT (2002) Tourismes 1 - Lieux communs, Paris : Belin, coll. Mappemonde. $320 \mathrm{p}$.

ESPACE TRANSSIBÉRIEN (2010) Transsibérien, Route de la soie, Saint Pétersbourg, Mongolie, Kamtchatka, catalogue 2009-2010, Espace EstOuest, Morges $1.16 \mathrm{p}$.
GOLDFARB, S. (2002) Listvianka, Irkoutsk : KP Baïkal. 144 p.

GOMEZ, Bruno (2010) «Le monde des voyagistes français. Palmarès Europe Centrale», Echo touristique, Hors-série octobre, p. 11-12.

GOSKOMSTAT - Comité d'État russe pour les statistiques (2010) Résultats du recensement de la population de la Fédération de Russie, Comité d'État russe pour les statistiques, vol. $1,<\mathrm{http}$ ://www.gks.ru/free_doc/new_site/ perepis2010/croc/perepis_itogil612.htm>, consulté le 12 novembre 2013.

GOUVERNEMENT DE LA RÉGION DE NOVOSSIBIRSK (2007) Arrêté $n^{\circ} 474$ : La Stratégie de développement socio-économique de l'oblast de Novossibirsk allant jusqu'en 2025, Novossibirsk : Ministère du développement économique de la région de Novossibirsk, 3 décembre.

GOUVERNEMENT DE LA RÉGION DE NOVOSSIBIRSK (2011) Programme fédéral Développement du tourisme dans la région de Novossibirsk pour la période 2012-2016, Novossibirsk : Ministère du développement économique de la région de Novossibirsk. 24 p. GOUVERNEMENT DE LA RÉGION DE NOVOSSIBIRSK (2013) Annexe 1 au programme fédéral Développement du tourisme dans la région de Novossibirsk pour la période 2012-2016, Novossibirsk : Ministère du développement économique de la région de Novossibirsk. 1 p.

INSEE - Institut national de la statistique et des études économiques (2004) «EPCV - Les vacances en $2004 »$, INSEE, <http://www.insee.fr/fr/themes/document.asp?ref_id=vac2004>, consulté le 5 novembre 2013.

INTERMÈDES (2011) «De Moscou à Pékin, le Transsibérien », <http:// www.intermedes.com/voyages/recherche? $\mathrm{w}=$ transsib\%C3\%A9rien\&x=1 $4 \& y=9>$, consulté le $1^{\text {er }}$ octobre 2012.

IRKUTSKMEDIA (2012) « [Le gouverneur de l'oblast d'Irkoutsk ne croit pas à l'arrivée des millions de touristes à Listvianka] », IrkutskMedia, $<$ http://irkutskmedia.ru/news/politics/16.11.2012/239961/ne-verit-vmillioni-turistov-na-baykale-v-listvyanke-gubernator-irkutskoy-oblasti. html >, consulté le 15 septembre 2013.

KOMMERSANT (2012) «Aux portes du Baïkal», Kommersant, <http:// www.kommersant.ru/vlast/74551>, consulté le 20 novembre 2013.

KUONI (2011) Émotions, catalogue 2011-2012, Travel lab, Saint Ouen, $<$ http://www.kuoni-emotions.com/emotions/intro.html>, consulté le $1^{\text {er }}$ octobre 2012.

LAUZANNE, Bertrand (2003) «Les Transsibériens», Regard sur l'Est, $<$ http://www.regard-est.com/home/breve_contenu.php?id=408>, consulté le 5 septembre 2012.

Loi fédérale $n^{\circ} 116-\mathrm{F} 3$ (2005) «Les Zones économiques spéciales en Fédération de Russie». Gouvernement de Russie. 34 p.

MONDOU, Véronique et Philippe VIOLIER (2009) «Projets, pratiques et lieux touristiques, quelles relations?», Mappemonde, vol. 24, $\mathrm{n}^{\circ}$ 2, p.1-15.

MOSSE, Claude (2001) Le Transsibérien, Paris : Plon. 350 p.

NOUVELLES FRONTIÈRES (2011) «Circuit Transsibérien, les contrées de l'infini », Nouvelles Frontières, <http://www.nouvelles-frontieres.fr/ voyages_circuits/voyages_circuits_russie/voyages-circuits-irkoutsk-circuit-transsiberien-les-contrees-de-linfini-RUSCTTRA-circuits.html>, consulté le $1^{\text {er }}$ octobre 2012.

POLINO, Marie-Noëlle (2007) «Patrimoine ferroviaire et tourisme : des relations anciennes et particulières ", Espaces, $n^{\circ} 251$, p. 26-31.

REMENCOV, Andrei (2003) Iz istorii sozdaniâ velikogo Transsibirskogo želežnodorojnogo puti [L'histoire de la création du grand chemin de fer Transsibérien], Moscou : Éditions de l'Université technique d'État. 170 p.

SALAÜN HOLIDAYS (2012) "Voyage dans le train des steppes», catalogue 2011-2012, Salaün Holidays, $<$ http://brochure.salaun-holidays. com/voyages.a.bord.du.transsiberien.voyage.dans.le.train.des.steppes-2-59-125-0.html\#125 >, consulté le 3 octobre 2012. 
SENIN, A. (2001) «Russkuû zemlû svâžet želežnij put'. Predystorâ Velikogo Sibirskogo Puti» [La terre russe sera liée par la voie de fer. La préhistoire de la Grande Voie Sibérienne], Inžener, Ingénieur, n 9 , p. 18-22.

SLAVENTURES (2011) Trains mythiques, Slaventures, catalogue 2011-2012, Nancy. 20 p.

STOCK, Mathis (sous la direction de) (2003) Le tourisme : acteurs, lieux et enjeux, Paris : Belin. 304 p.

THOMAS, Albert (2005) Le Transsibérien, Magellan et cie, collection Heureux qui comme... $85 \mathrm{p}$.

UHTOMIN, M.I. (2005) Transsibirskaâ magistral' [Le Transsibérien], Moscou : Prior. 160 p.
VIOLIER, Philippe (2011) «Les lieux du monde», Espaces Temps, $<$ http://www.espacestemps.net/articles/les-lieux-du-monde/>, consulté le 15 septembre 2012.

VIOLIER, Philippe; Mathis STOCK et Philippe DUHAMEL (2009) "Variations d'altérité et "fin" du tourisme». Colloque Fins et confins du tourisme. Grenoble. 26-27 mai.

VOYAGEURS DU MONDE (non daté) "Nos voyages "Transsibérien”", Voyageurs du monde, <http://www.voyageursdumonde.fr/voyage-surmesure/recherche-voyage-tag/voyage-transsiberien>, consulté le $1^{\text {er }}$ octobre 2012.

\section{ANS D'EXPÉRIENCE EN TOURISME Complétez votre collection}

$\square$ Tourisme cynégétique et halieutique Tourisme des routards

vol. 32, $n^{0} 1,2013$

$\square$ Le tout-inclus vol. $31, n^{0} 2,2012$

$\square$ Gouvernance des parcs au Nunavik vol. 31, no 1, 2012

$\square$ Tourisme et patrimoine mondial Les associations de patrimoine et le tourisme vol. 30, $n^{0} 2,2011$

$\square$ Excellence des destinations Ciné-tourisme vol. 30, n ${ }^{0} 1,2011$

$\square$ Géotourisme / Tourisme et femmes vol. $29, n^{0} 2,2010$

$\square$ Tourisme des racines Tourisme et autochtones vol. $29, n^{0} 1,2010$

$\square$ Tourisme, sport et développement vol. $28, n^{0} 2,2009$ $\square$ Tourisme polaire vol. 28, no 1, 2009

$\square$ Nouveaux musées, nouveaux tourismes vol. $27, n^{0} 3$, automne 2008

$\square$ Les grands équipements touristiques vol. $27, n^{\circ} 2$, été 2008

$\square$ La science du tourisme vol. $27, n^{0} 1$, printemps 2008

$\square$ Tourisme et solidarité vol. 26, n 3, automne 2007

$\square$ Tourisme et attractivité vol. 26, n² 2, été 2007

$\square$ Tourisme Caraïbe vol. $26, n^{0} 1$, printemps 2007

$\square$ Forêt vol. $25, n^{\circ} 3$, automne 2006

$\square$ Désirs d'Orient vol. 25, n² 2, été 2006

$\square$ Tourisme gourmand vol. $25, n^{0} 1$, printemps 2006 $\square$ Tourisme et santé vol. $24, n^{\circ} 3$, automne 2005

$\square$ Tourisme, religion et patrimoine vol. 24, nº 2, été 2005

$\square$ Maroc

vol. 24, no 1, printemps 2005

$\square$ Regards sur l'hôtellerie vol. 23, n ${ }^{\circ} 3$, automne 2004

$\square$ La qualité en tourisme vol. 23, n² 2, été 2004

$\square$ Au risque du politique vol. $23, n^{0} 1$, printemps 2004

$\square$ L'accessibilité, une conquête inachevée vol. 22, n³ 3, automne 2003

$\square$ Les routes à thèmes vol. 22, n² 2, été 2003

$\square$ Tourisme et sexualité vol. 22, $n^{0} 1$, printemps 2003

\section{$20 \$$ / numéro, taxes incluses. Livraison gratuite pour tout achat de plus de $50 \$$.}

\section{Faites vos achats sur www_coopugam.com.}

